\title{
Metabotropic Glutamate Receptor 5 Upregulation in A-Fibers after Spinal Nerve Injury: 2-Methyl-6-(Phenylethynyl)-Pyridine (MPEP) Reverses the Induced Thermal Hyperalgesia
}

\author{
Lindsey J. Hudson, ${ }^{3}$ Stuart Bevan, ${ }^{1}$ Kara McNair, ${ }^{1}$ Clive Gentry, ${ }^{1}$ Alyson Fox, ${ }^{1}$ Rainer Kuhn, ${ }^{2}$ and \\ Janet Winter ${ }^{1}$ \\ ${ }^{1}$ Novartis Institute for Medical Sciences, London, WC1E 6BS, United Kingdom, ${ }^{2}$ Novartis Pharma AG, Werk Klybeck, \\ CH-4057 Basel, Switzerland, and ${ }^{3}$ Oxford GlycoSciences Ltd., Abingdon, OX14 4RY, United Kingdom
}

Metabotropic glutamate receptor 5 (mGluR5) protein increased after sciatic nerve section in ipsilateral L4 and L5 DRG neuronal profiles, with most of the increase occurring in myelinated A-fiber somata. mGluR5 also increased in lamina II of the ipsilateral spinal cord and the proximal sciatic nerve stump in this model. After L5 spinal nerve ligation, mGluR5 immunoreactivity increased dramatically not only in damaged L5 but also in the neighboring undamaged L4. Interestingly, after partial sciatic nerve section, mGluR5 expression did not change in either L4 or L5 DRG neuronal profiles.

Both spinal nerve ligation and sciatic nerve partial section produced significant mechanical and thermal hyperalgesia and tactile allodynia. After partial sciatic nerve section, the mGluR5specific antagonist 2-methyl-6-(phenylethynyl)-pyridine (MPEP) had no effect on any of these behaviors. However, after L5 spinal nerve ligation, although MPEP failed to alter the induced tactile allodynia or mechanical hyperalgesia, it dose dependently reversed the developed thermal hyperalgesia.

Therefore, reversal of thermal hyperalgesia by MPEP correlates with increased mGluR5 in lumbar DRG A-fiber somata after nerve injury. Furthermore, A-fibers in the uninjured L4 DRG after L5 spinal nerve ligation that have increased mGluR5 are the same A-fibers that newly express vanilloid receptor 1 after such injury. Together, these results suggest that, after L5 spinal nerve injury, mGluR5 expression on A-fibers is essential to the development of thermal hyperalgesia. After partial nerve section, however, it is unlikely that thermal responses are mediated through mGluR5 because no such increase in mGluR5 is detected in this model and MPEP is ineffective.

Key words: MPEP; mGluR5; nerve injury; upregulation; thermal hyperalgesia; VR1
Glutamate is the most widespread neurotransmitter in the mammalian CNS and has an established role in the transmission of acute and chronic pain (Urban and Gebhart, 1999). The actions of glutamate are mediated through ligand-gated ion channels (ionotropic glutamate receptors) and G-protein-coupled metabotropic glutamate receptors (mGluRs) (Baranauskas and Nistri, 1998). mGluRs are divided into three groups, with growing evidence that group I mGluRs, i.e., mGluR1 and mGluR5, are important in nociception and pain behaviors after tissue injury (Dorgul et al., 2000; Walker et al., 2001a,b; Bhave et al., 2001).

mGluR5 protein is present in laminae I and II of the spinal cord in which nociceptive primary afferents terminate (Valerio et al., 1997a,b). Furthermore, in normal rats, mGluR5 protein is constitutively expressed on a subpopulation of dorsal root ganglion (DRG) neurons, some of which are nociceptors determined by a substantial overlap with vanilloid receptor 1 immunoreactivity (VR1-IR) and in peripheral nerve fibers in skin (Walker et al., 2001b).

There is much evidence to suggest that peripherally expressed mGluR5 is important in nociception. Indeed, in normal mice,

\footnotetext{
Received July 23, 2001; revised Dec. 10, 2001; accepted Jan. 11, 2002.

We thank Katherine Walker for helpful discussions.

Correspondence should be addressed to Janet Winter, Novartis Institute for Medical Sciences, 5 Gower Place, London WC1E 6BS, UK. E-mail: janet.winter@ pharma.novartis.com.

K. McNair's present address: Department of Neuroscience and Biomedical Systems, University of Glasgow, Glasgow, G12 8QQ, UK.

Copyright (C) 2002 Society for Neuroscience $0270-6474 / 02 / 222660-09 \$ 15.00 / 0$
}

peripheral injection of a group I agonist dihydroxyphenylglycine increased thermal sensitivity, which was abolished by pretreatment with either the mGluR5 selective antagonist 2-methyl6-(phenylethynyl)-pyridine (MPEP) (Gasparini et al., 1999) or the mGluR1 antagonist LY367385 (Bhave et al., 2001). Furthermore, in the formalin model of inflammatory pain, peripherally applied group 1 antagonists MPEP and [7-(hydroxy-imino) cyclopropa[b]chromen-1a-carboxylate ethyl ester] (CPCCOEt) also blocked thermal hyperalgesia (Bhave et al., 2001), and MPEP blocked the Freund's complete adjuvant (FCA)-induced mechanical hyperalgesia (Walker et al., 2001a). Interestingly, centrally administered MPEP (intrathecal or intracerebroventricular) failed to affect the FCA-induced mechanical hyperalgesia, further supporting the role of peripheral mGluR5 receptors in hyperalgesia.

After partial ligation of the sciatic nerve, the mGluR5 antagonist MPEP did not significantly reverse mechanical hyperalgesia (Walker et al., 2001a). Dogrul et al. (2000) found that spinal or local administration of the mGluR5 antagonist SIB-1757 in rat was weakly active against the tactile allodynia that developed $5 \mathrm{~d}$ after L5 spinal nerve ligation. Interestingly, in this study, SIB1757 resulted in a complete reversal of the induced thermal hyperalgesia (Dogrul et al., 2000).

Several studies have examined the role of mGluR5 in nerve injury-induced hyperalgesia, with a specific role suggested for mGluR5 in the mediation of thermal hyperalgesia. However, different pain models, behavioral tests, and antagonists were used in each study. Moreover, to our knowledge, there are no reports 
in the literature that measure changes in mGluR5 expression in DRG neurons in different models of pain and correlate these changes with the injury-induced behavior.

In this study, using anatomical separation or fluorescent tracers to distinguish the somata of damaged or intact fibers, we examined mGluR5-IR in L4 and L5 DRG neurons in three models of nerve injury. In two of these models, we performed behavioral tests to show the development of significant thermal and mechanical hyperalgesia, as well as tactile allodynia. We correlated the effectiveness of the mGluR5 antagonist MPEP in reversing the thermal hyperalgesia with increased mGluR5 expression in the DRG from these models.

\section{MATERIALS AND METHODS}

Animals and surgical methods. Adult male Wistar rats (120-140 gm body weight; Charles River, Cardiff, UK) were used in this study; 6-12 animals were used per group for behavioral testing and three per group for immunohistochemical analysis. All surgical procedures were performed under enflurane $-\mathrm{O}_{2}$ inhalation anesthesia according to United Kingdom Home Office approved procedures.

Partial sciatic nerve section and introduction of fluorescent tracer. The methodology for partial sciatic nerve section and fluorescent tracer application to locate the damaged and intact neuronal populations has been described previously in detail (Hudson et al., 2001). Briefly, the left sciatic nerve was exposed at midthigh level, and approximately half the width of the nerve was carefully isolated and sectioned. The distal end of the section was tightly tied with $7 / 0$ silk. The cut fibers at the proximal end were carefully separated from intact fibers for a length of $\sim 5 \mathrm{~mm}$ and placed into the opening of a vessel filled with FluoroRuby (FR) [10\% solution (w/v) in sterile saline; Molecular Probes, Eugene, OR] for 10 min, after which the cut end was ligated with $7 / 0$ silk. At the same time as the surgery, $101 \mu \mathrm{l}$ intradermal injections of fast blue (FB) $(1 \% \mathrm{w} / \mathrm{v}$ dissolved in sterile water; Sigma, Poole, UK) were administered at the glabrous hairy border of the left hindpaw. The FB-labeled somata correspond to the intact axons, whereas the FR-labeled somata correspond to the damaged axons. We showed in a previous study that these two tracers are specific and that they do not overlap (Hudson et al., 2001). Furthermore, we also showed previously that the presence of the tracer has no effect on the staining intensity of the other fluorescent channels used (Hudson et al., 2001). FB injections were also administered to the left hindpaw of naïve rats to locate the target-specific afferent population. These injections were conducted under light anesthesia as described above. Partial sciatic nerve-ligated animals, both with and without fluorescent tracer introduction, were left to recover for 2 weeks before behavioral tests, after which tissue samples were removed for analysis.

Total section of the sciatic nerve. Following the same methodology described above, the sciatic nerve was isolated at midthigh level and completely sectioned, and a $1 \mathrm{~cm}$ portion of nerve was removed. The two ends of the sciatic nerve were then tightly ligated with $7 / 0$ silk to prevent regeneration. Tissue samples were taken 2 weeks after the surgery.

Spinal nerve ligation (Chung model). Ligation of the left L5 spinal nerve was performed following the methods of Kim and Chung (1992). Anesthetized rats were placed into a prone position, and an incision was made to the left of the spine at the L4-S2 level. A deep dissection was made through the paraspinal muscles, which were then separated from the spinal processes at the L4-S2 level to reveal the sciatic nerve as it branches to form the L4, L5, and L6 spinal nerves. The L6 transverse process was carefully removed, allowing visualization of L5 and L6 spinal nerves. The L5 spinal nerve was then isolated and tightly ligated with silk suture. In sham-operated animals, the same surgical procedure was performed except for ligation of the L5 spinal nerve. The wound was closed with sutures and clips and dusted with antibiotic powder. Animals were left to recover for 2 or 3 weeks before behavioral testing.

Drugs and behavioral testing for pain. Mechanical hyperalgesia was assessed by measuring paw withdrawal thresholds to an increasing pressure stimulus placed onto the dorsal surface of the paw using an analgesymeter (model 7200; Ugo Basile, Milan, Italy) with a cutoff of $250 \mathrm{gm}$. Withdrawal thresholds were measured on both the ipsilateral (ligated) and contralateral (unligated) paw before (predose) and then $3 \mathrm{hr}$ after drug or vehicle administration. Data are expressed as withdrawal threshold (in grams).

Thermal hyperalgesia was examined by measuring the latency to withdrawal of the hindpaws from a focussed beam of radiant heat applied to the plantar surface using a Ugo Basile Plantar Test apparatus. Two days before the experiment, animals were placed in a transparent Perspex box with a thin glass floor and allowed to acclimatize for 10-15 min before withdrawal latencies were measured on both ipsilateral and contralateral paws. On the day of the experiment, withdrawal latencies were measured immediately before and then $3 \mathrm{hr}$ after oral administration of MPEP (10 or $100 \mathrm{mg} / \mathrm{kg}$ ) or vehicle. An additional group of age-matched naïve animals was included as a positive control. On all three occasions, the withdrawal latency of each paw was measured twice with a 5 min interval, and the mean of the two readings was used for data analysis. With each reading, the apparatus was set with a cutoff time of $23 \mathrm{sec}$.

Tactile allodynia was assessed by measuring withdrawal thresholds to calibrated von Frey hairs with intensities ranging from 1 to $20.9 \mathrm{gm}$. Filaments exerting a force above $20.9 \mathrm{gm}$ were not used because they produced lifting of the paws. On the day of the experiment, animals were placed in a Perspex chamber with mesh metal floor and allowed to acclimatize for 15-30 min. Starting with the lowest filament force, von Frey hairs were applied perpendicular to the midplantar surface of both hindpaws, with sufficient force to cause slight bending against the paw and held for a few seconds. This was repeated five times with an interval of 1-2 sec. A positive response was noted if the paw was sharply withdrawn or there was flinching during removal of the hair. If no response was noted to any trial, the process was repeated with the next higher force hair and the filament that produced a positive response was denoted as the threshold. At least 5 min was left between trials with successive filaments. Withdrawal thresholds were determined before and $3 \mathrm{hr}$ after administration of MPEP $(10$ and $100 \mathrm{mg} / \mathrm{kg})$ or vehicle. An additional group of age-matched naïve animals was included as a positive control. Behavioral data were compared using ANOVA with Tukey's multiple comparisons post hoc test. Each experiment for measuring either thermal hyperalgesia or allodynia had six animals per group. Where experiments were repeated, data from the separate experiments were combined as indicated in the figure legends.

MPEP was synthesized as described previously by Gasparini et al. (1999) and made up in $0.5 \%$ methyl cellulose. Drugs were administered orally in a volume of $1 \mathrm{ml}$ at 10 or $100 \mathrm{mg} / \mathrm{kg}$.

Tissue preparation. Fourteen or $21 \mathrm{~d}$ after surgery and/or fluorescent tracer application, the animals were killed by $\mathrm{CO}_{2}$ asphyxiation, and lumbar L4 and L5 DRG neurons, as well as spinal cord and SCG, were dissected out from both ipsilateral and contralateral sides. Samples were mounted in Tissue Tek O.C.T. embedding compound (Raymond A. Lamb, Eastbourne, UK), quickly frozen on dry ice, and then stored at $-80^{\circ} \mathrm{C}$. DRGs were sectioned using a Bright cryostat to $10 \mu \mathrm{m}$ thickness, whereas nerves were cut to a thickness of $30 \mu \mathrm{m}$. Tissue sections were thaw mounted onto Superfrost polysine-coated slides (BDH Laboratory Supplies, Dorset, UK) and air dried for $30 \mathrm{~min}$. Slides were then stored at $-80^{\circ} \mathrm{C}$ before use. Sections of DRG neuron labeled with the fluorescent tracers were imaged before fixation because fixing was found to diminish the fluorescence. A Nikon (Tokyo, Japan) Eclipse 800 fluorescent microscope, with the relevant filters, attached to a Hamamatsu (Shizouka, Japan) cooled CCD camera, and Image Pro Plus software (Media Cybernetics, Silver Spring, MD) was used. The sections were then processed for immunocytochemistry as outlined below.

Before immunostaining, sections were fixed in $4 \%$ paraformaldehyde for $30 \mathrm{~min}$, washed in PBS, and then blocked with $10 \%$ normal donkey serum $(30 \mathrm{~min})$. Sections were then incubated in a combination of primary antibodies overnight [affinity-purified rabbit anti-mGluR5, 1:1000, fully characterized by Valerio et al. (1997b); mouse antineurofilament clone (N52), 1:10,000 (Sigma); guinea pig anti-VR1, 1:500 (Chemicon, Temecula, CA); diluted in PBS, $0.1 \%$ Triton X-100, and $0.002 \%$ sodium azide) with biotinylated isolectin B4 (IB4) (final concentration of $5 \mu \mathrm{g} / \mathrm{ml}$; Sigma) added to the mixture in some cases. Sections were then incubated in the relevant mixture of donkey anti-rabbit, donkey anti-mouse, or donkey anti-guinea pig antibodies (Jackson ImmunoResearch, West Grove, PA) conjugated to FITC, tetramethylrhodamine isothiocyanate, or 7-amino-4-methylcoumarin-3-acetic acid (AMCA) and/or streptavidin AMCA (1:500; Sigma) secondary reagents diluted in antibody buffer for $1 \mathrm{hr}$. All incubations were performed in a humidified chamber at room temperature. Antibody-stained DRG sections were imaged, and the backlabeled cells were classified as positive or negative for FR and FB by matching the profiles with the images taken before antibody staining. mGluR5 and N52 staining intensity was quantified manually using Image Pro Plus image analysis software (Media Cybernetics). All samples from a single experiment were processed for 
immunohistochemistry in the same session, and images were captured using identical conditions to allow accurate comparisons to be made. Because of the variable nature of immunohistochemistry, samples from different experiments cannot be compared because staining intensity can vary between experiments.

Statistical analysis and data presentation. Controls in which the primary antibody was replaced with antibody buffer were conducted alongside each experiment for each of the tissues examined. The measured immunofluorescences of the DRG neurons in such controls were normally distributed. In contralateral and naïve tissues that were stained for mGluR5, a population of cells with a similar normal distribution of low-level fluorescence (to that of the no primary antibody control group) was present, as well as a second group of cells with higher levels of mGluR5-IR. The mean fluorescence plus 2 SDs of the contralateralnaïve mGluR5-negative populations was used as the cutoff point for positive staining within a particular experiment (Hudson et al., 2001). The mean intensity of the mGluR5-negative population was set to 1, with the rest of the data within the same experiment normalized to this control value. Neuronal fluorescence was measured every 10 th section of DRG to prevent the same neuron being measured twice. All neurons in three sections were measured per ganglion, except for neurons that were incomplete at the edge of the section that were excluded using a frame. This way, 800-1000 DRG neurons were measured per DRG examined. Data from experimental and control populations were compared statistically using Kruskal-Wallis ANOVA with Dunn's multiple comparisons post hoc test. Statistical differences were similar for the grouped data (data pooled from three animals) and for each of the individual data sets.

Rather than using mean fluorescence intensity to compare different populations, which can be misleading, summary data were presented as a "population intensity index" (PI). This measure is calculated as follows: population intensity index $=$ percentage of total population measured $\times$ mean fluorescence.

As well as including mean fluorescence, this measurement also accounts for the proportion of the total neurons measured that are in the specific population of interest.

The PI indices, for both negatively and positively stained populations across-between experimental and control groups, were compared using Fisher's exact test.

\section{RESULTS}

The majority of the mGluR5-stained neuronal profiles in L4 and L5 DRG from naïve rats did not counterstain for neurofilament (a marker of myelinated A-fibers). Furthermore, the mean crosssectional area of the mGluR5-positive neuronal profiles was 359 $\mu \mathrm{m}^{2}$, which puts them in the small-diameter C-fiber class. We double labeled the sections with IB4 (a marker of nonpeptidergic C-fibers), and this population accounted for the majority of the mGluR5-positive profiles (data not shown).

\section{Complete sciatic nerve section: immunohistochemistry}

Two weeks after complete sciatic nerve section, mGluR5-IR had increased in the proximal sciatic nerve stump, in which staining was more extensive and on finer fibrillar structures, presumably axons compared with control nerve (Fig. 1a,b). Lumbar 4 and 5 sections of spinal cord from this model were triple labeled with mGluR5, IB4, and N52 (Fig. 1c-e). mGluR5-IR was much greater in ipsilateral lamina II inner compared with the contralateral side (Fig. 1c) and was strong in the region of IB4 loss in ipsilateral lamina II after the axotomy (Fig. 1c,e). In the overlaid mGluR5and N52-stained images, some overlap of mGluR5 and N52 was visible in lamina III ipsilateral spinal cord (Fig. $1 f, g$ )

In ipsilateral L4 and L5 DRG neurons, PI index for mGluR5-IR increased significantly compared with contralateral and naïve DRG $(p<0.001)$ (Fig. $2 a-f)$. Indeed, in this experiment, both the mean mGluR5-IR [intensity $(i)$ ] and the percentage of neurons measured that were positive increased in ipsilateral DRG neurons $(i, 1.91 \pm 0.019 ; 87 \%)$ compared with naïve $(i$, $1.73 \pm 0.013 ; 66 \%)$ and contralateral $(i, 1.85 \pm 0.015 ; 60 \%)$ DRG.

Using an anti-neurofilament counterstain, it was found that the
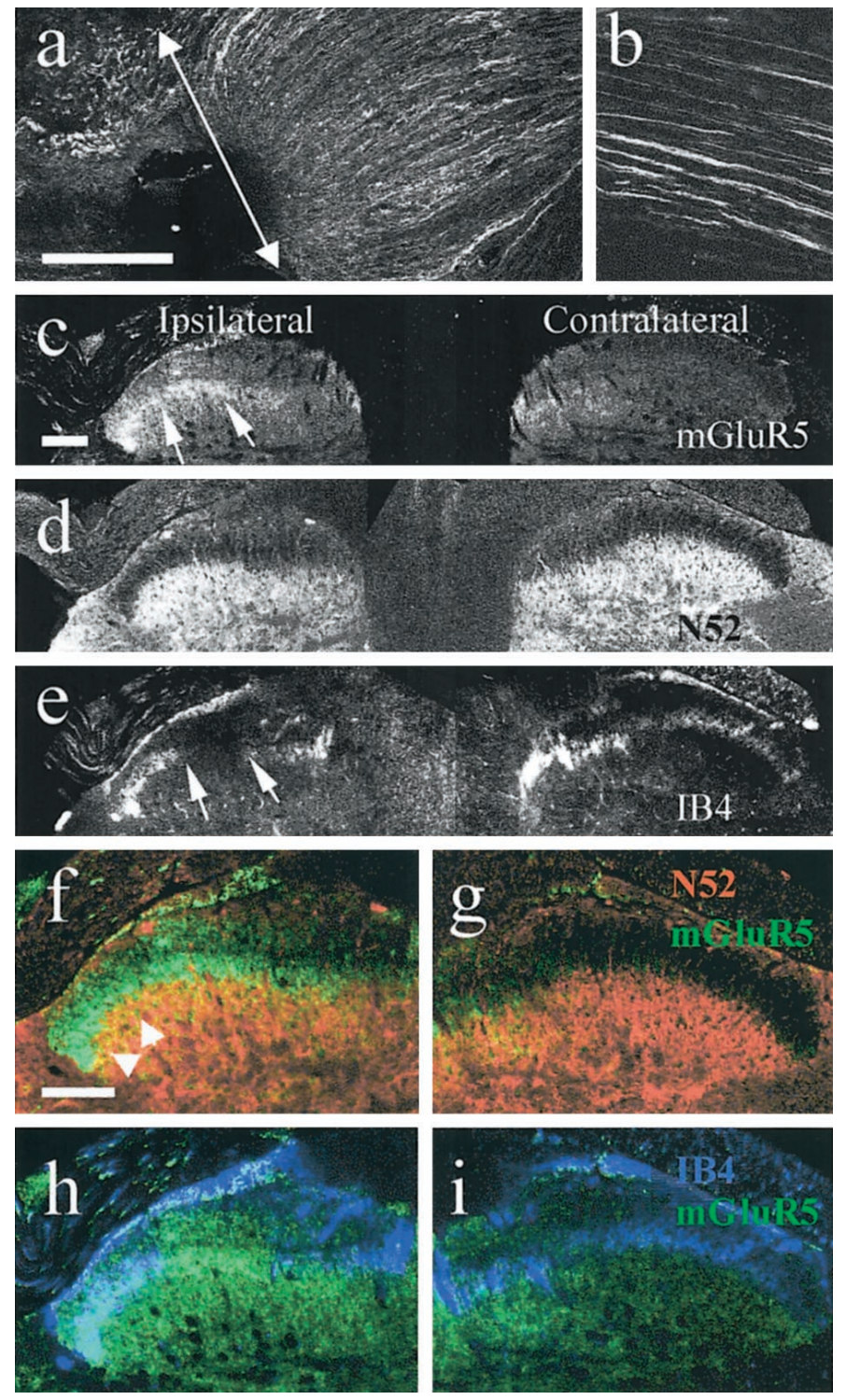

Figure 1. mGluR5 expression in 30- $\mu$ m-thick sections of sciatic nerve and spinal cord 2 weeks after sciatic nerve axotomy. $a$, An accumulation of mGluR5 expression was detected in the sciatic nerve stump proximal to the lesion (arrow) when compared with sciatic nerves from naive rats $(b)$. $c$, mGluR5 expression levels were also increased in the ipsilateral lamina II of the spinal cord (arrows) 2 weeks after sciatic nerve axotomy. $d$ and $e$ are the same section as in $c$ stained with anti-neurofilament and IB4, respectively. Arrows in $c$ and $e$ indicate the ipsilateral loss of IB4 staining, which is typical after sciatic nerve axotomy, and the corresponding increase in mGluR5 expression in this region. $f$ and $g$ are higher-power images, showing N52 staining in red and mGluR5 staining in green. In lamina III, some overlap (orange-yellow) is apparent in the ipsilateral side. $h$ and $i$ are the same sections as in $f$ and $g$ stained for mGluR5 (green) and IB4 (blue). Scale bars: $a, b, 200 \mu \mathrm{m} ; c-i, 500 \mu \mathrm{m}$.

majority of the changes measured in mGluR5 expression were in the ipsilateral A-fiber population, which showed an approximately threefold increase in PI index $(p<0.001)$ (Fig. $2 g)$. This is illustrated in the scatter plots, which show increases in mGluR5 expression levels in the somata with medium to large crosssectional areas (Fig. 2a-c).

\section{Partial sciatic nerve section: immunohistochemistry}

Two weeks after fluorescent tracer introduction and sciatic nerve partial section, no significant differences were detected in 


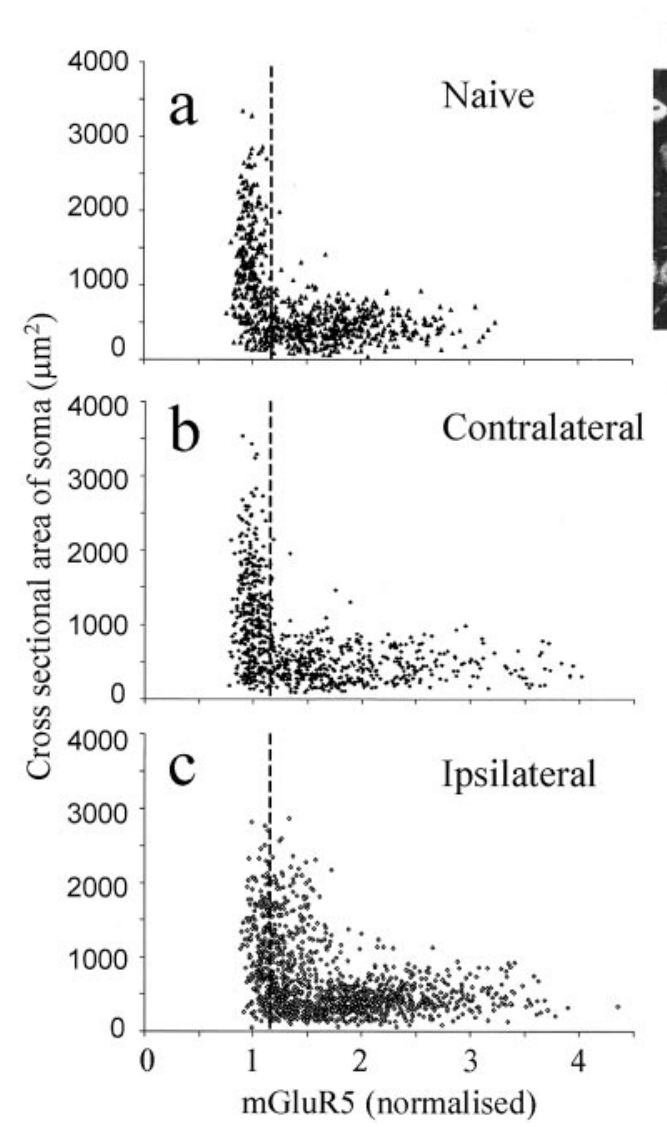

Contralateral
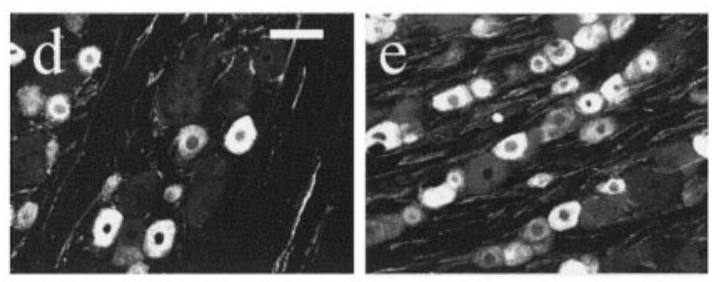

$\mathrm{f}$

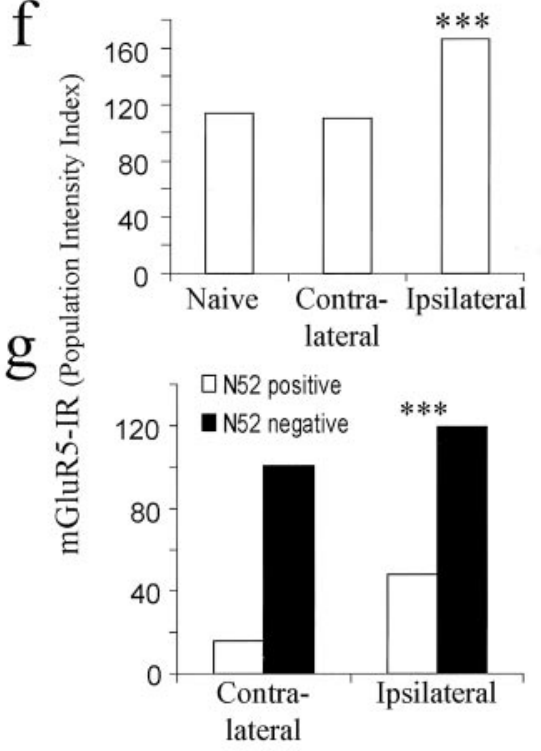

Figure 2. Two weeks after sciatic nerve section, mGluR5-IR and somata cross-sectional area were plotted from naïve $(a)$, contralateral $(b)$, and ipsilateral (c) DRG. A clear shift in mGluR5 expression levels can be seen in ipsilateral DRG, especially in those neurons with larger somata cross-sectional areas. This increase in mGluR5-IR in ipsilateral DRG neurons is illustrated in $d$ and $e$. $f$ shows the PI of the total neuronal populations in the 3 groups. The PI of ipsilateral DRG is $40 \%$ greater than that of either contralateral or naïve DRG neurons $(p<0.001) . g$, These changes in mGluR5 expression were examined in more detail by counterstaining with anti-neurofilament to separate out the A- and C-fiber neuronal somata. The majority of the increase seen in ipsilateral DRG neurons was found to be in the A-fiber population (N52-positive), with only small increases seen in the neurofilament-negative population. Scale bar, $100 \mu \mathrm{m}$. Data were compared using Fisher's exact test; ${ }^{* * *} p<0.001$.

mGluR5-IR PI index or in intensity or percentage of positively stained neurons between ipsilateral $(i, 1.55 \pm 0.018 ; 61 \%)$, contralateral $(i, 1.67 \pm 0.017 ; 53 \%)$, or naïve $(i, 1.59 \pm 0.015 ; 62 \%)$ DRG neuronal profiles (Fig. 3). Using fluorescent retrograde tracers, the somata with damaged axons (FR, introduced to the transected nerve) and those with intact axons (FB, injected into the paw) were examined separately. mGluR5-IR PI, intensity, and the percentage of positively classified neurons decreased slightly in the damaged population $(i, 1.33 \pm 0.035 ; 47 \%)$ compared with intact $(i, 1.42 \pm 0.031 ; 50 \%)$ or control $(i, 1.41 \pm 0.025$; $54 \%$ ) populations (Fig. $4 a-c, e)$.

Interestingly, mGluR5 expression levels were increased in the proximal damaged portion of the sciatic nerve (Fig. $4 d$ ).

\section{Partial sciatic nerve section: behavior}

Two weeks after partial sciatic nerve section, animals exhibited thermal hyperalgesia of the ipsilateral paw (Fig. 5). There was no significant change in withdrawal latencies of contralateral paws or hindpaws from sham-operated rats.

Oral administration of MPEP (10 and $100 \mathrm{mg} / \mathrm{kg}$ ), the specific mGluR5 antagonist, did not affect the paw withdrawal latencies in naïve or sham-operated animals and failed to reverse the thermal hyperalgesia caused by the partial nerve section (Fig. 5). Previously, we reported a lack of reversal of mechanical hyperalgesia or allodynia by MPEP in the same model (Walker et al., 2001a).

\section{L5 spinal nerve ligation: immunohistochemistry}

Three weeks after sham surgery, mGluR5 expression levels in both the A- and C-fiber populations were not significantly different from those of DRG from naïve or contralateral animals (data not shown). Ether 2 or 3 weeks after L5 spinal nerve ligation, however, mGluR5 expression had increased in both uninjured L4 and injured L5 ipsilateral DRG neuronal profiles compared with contralaterals (Fig. $6 a-d$, only 3 week data shown). Increases in both mean mGluR5-IR and percentage of positively classified DRG neurons were measured in intact L4 ( $i, 1.9 \pm 0.013 ; 74 \%)$ and damaged L5 $(i, 1.95 \pm 0.014 ; 87 \%)$ ipsilateral DRG neurons compared with contralateral DRG $(i, 1.56 \pm 0.016 ; 55 \%)$. Using an anti-neurofilament counterstain to detect myelinated A-fibers, mGluR5 PI was found to increase in both L4 and L5 ipsilateral A-fibers compared with controls. Large increases were measured in both the mean intensity and percentage of mGluR5-positive neurons of the ipsilateral L4 $(i, 1.88 \pm 0.015 ; 38 \%)$ and L5 ( $i$, $1.82 \pm 0.014 ; 57 \%)$ A-fiber populations when compared with controls $(i, 1.27 \pm 0.013 ; 6.1 \%)$. Unexpectedly, little change in nonmyelinated C-fiber mGluR5-IR was detected in ipsilateral L4 $(i, 1.93 \pm 0.015 ; 35.5 \%)$ or L5 $(i, 2.1 \pm 0.017 ; 38 \%)$ compared with controls $(i, 1.6 \pm 0.015 ; 49 \%)$.

VR1 and mGluR5 expression were measured in L4 ipsilateral and contralateral DRG neuronal profiles 3 weeks after L5 spinal 


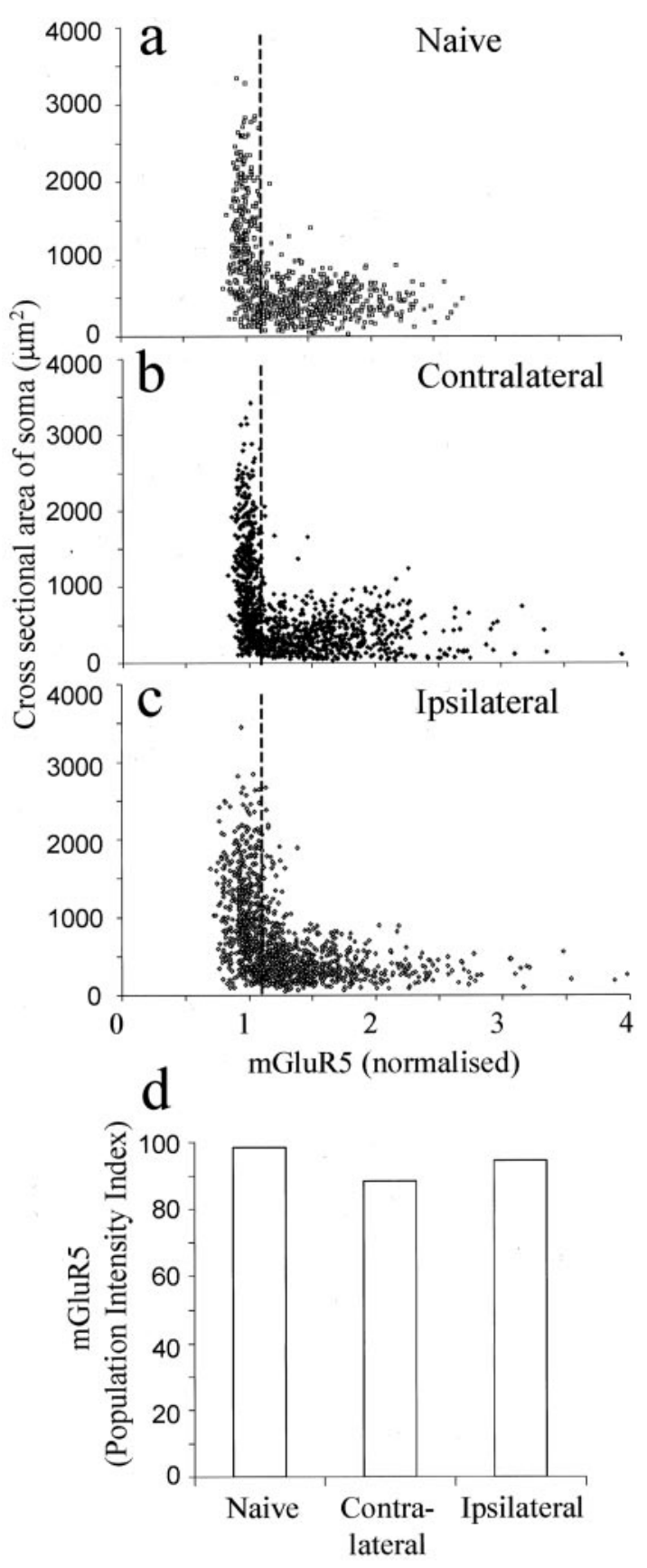

Figure 3. Changes in mGluR5 expression 2 weeks after partial sciatic nerve section. $a-c$, Scatter plots illustrating mGluR5-IR and somata cross-sectional area in naïve, contralateral, and ipsilateral L4 and L5 DRG neurons, respectively. $d$, PI of all neurons measured. Data were compared using Fisher's exact test, and no significant differences were found between naïve, contralateral, or ipsilateral DRG neurons.

nerve ligation (Fig. 7). Neurons were categorized as being neurofilament positive or negative using mouse anti-N52 counterstain. In contralateral L4 DRG neurons, 3\% of neurofilamentpositive profiles have positive VR1-IR. However, after L5 spinal nerve ligation, this increases to $33 \%$ in L4 ipsilateral DRG neurons (Fig. 7). Furthermore, $72 \%$ of these A-fibers that newly express VR1 after spinal nerve injury are also now positively IR for mGluR5 (Fig. 7).

\section{L5 spinal nerve ligation: behavior}

L5 spinal nerve-ligated rats exhibited a significant ipsilateral mechanical hyperalgesia, measured as a reduction in paw pressure withdrawal thresholds, 3 weeks after surgery, with no change in thresholds of contralateral paws or in sham-operated animals (Fig. 8a).

L5 spinal nerve-ligated animals also showed tactile allodynia, measured with von Frey hairs (Fig. 8b), and thermal hyperalgesia indicated by a reduction in hindpaw withdrawal latency (Fig. $8 c$ ) compared with naïve and contralateral withdrawal latencies and with sham-operated animals. Again, with both modalities, there was no change in withdrawal thresholds of contralateral paws or sham-operated animals (Fig. 8b,c).

Oral administration of MPEP (10 and $100 \mathrm{mg} / \mathrm{kg}$ ) did not affect the mechanical hyperalgesia (Fig. $8 a$ ) and caused only a slight reversal of the observed tactile allodynia, which was nonsignificant (Fig. 8b). However, it produced a significant reversal of the thermal hyperalgesia back to naïve control levels (Fig. $8 c$ ). Thus, after $100 \mathrm{mg} / \mathrm{kg}$, ipsilateral withdrawal latencies were not significantly different from either predose readings or those in naïve or sham-operated rats. MPEP at $10 \mathrm{mg} / \mathrm{kg}$ was also effective, such that ipsilateral latencies did not differ significantly from naïve and sham readings, although they were not significantly different from predose readings (Fig. 8c).

\section{DISCUSSION}

The mechanisms contributing to neuropathic pain include phenotypic changes in DRG neurons, anatomical rearrangement, e.g., central sprouting of A-fibers into lamina II of the dorsal horn (Woolf et al., 1992) and sympathetic sprouting (Lee et al., 1998), as well as possible death of inhibitory interneurons (PetersenZeitz and Basbaum, 1999). There is also controversy over the relative contribution of intact or damaged neuronal populations after partial nerve injuries in the development of neuropathic pain (for review, see Gold, 2000). In this study, we concentrated on rat models of neuropathic pain in which the damaged and intact DRG neuronal populations can be identified and analyzed separately. Furthermore, we used neuronal markers, e.g., antineurofilament, to distinguish between A- and C-fibers to further demarcate the populations.

We investigated the role of mGluR5 in neuropathic pain by correlating changes in expression levels with the ability of the specific antagonist MPEP to inhibit hyperalgesia. We found that, 2 weeks after total sciatic nerve section, mGluR5 increased in L4 and L5 DRG neuron cell bodies, primarily in A-fibers. Moreover, there was an increase in mGluR5-IR in laminas II and III in ipsilateral spinal cord, especially in the region of IB4 loss, as well as in the proximal sciatic nerve stump. Two weeks after partial sciatic nerve section, however, no changes were detected in mGluR5-IR in the somata of either the damaged or intact neuronal populations, which were identified by backlabeling with fluorescent neuronal tracers. Interestingly, mGluR5-IR did increase in the proximal damaged portion of the sciatic nerve. This has been seen previously with ion channels [VR1 and certain sodium channels (Hudson et al., 2001)], even when levels decrease in the cell bodies, and has been interpreted as a protein redistribution. Significant mechanical and thermal hyperalgesia, as well as allodynia, develop in the ipsilateral paws of these partial sciatic nerve-sectioned animals. Previously in this model, we reported a lack of reversal of mechanical hyperalgesia or allodynia by MPEP (10 or $100 \mathrm{mg} / \mathrm{kg}$, p.o.) (Walker et al., 2001a), and, in the present 


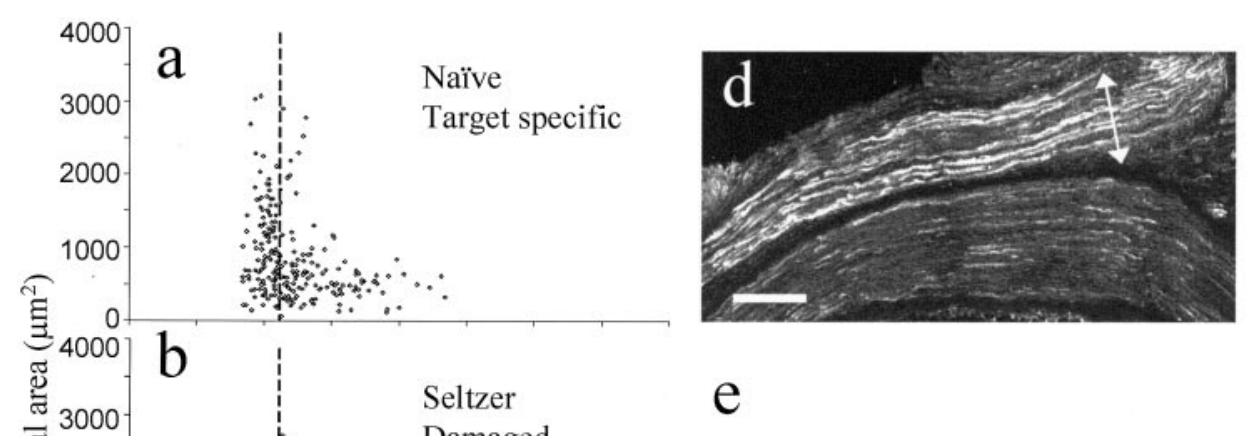

Damaged
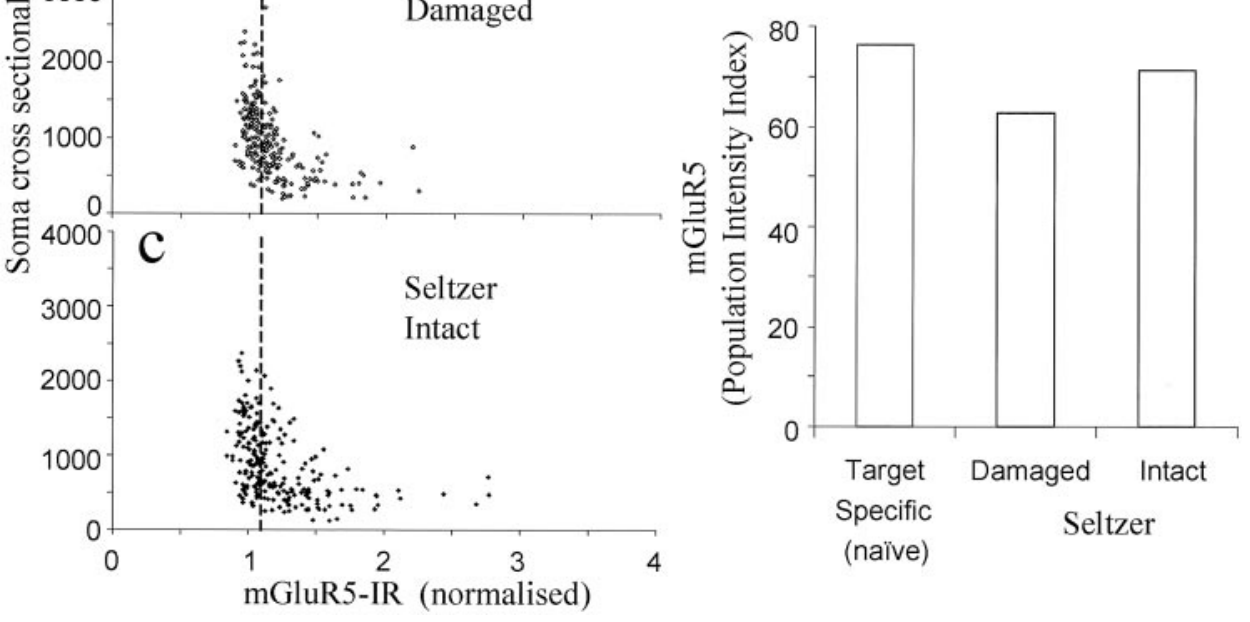

Figure 4. mGluR5 expression in the damaged and intact neuronal populations after partial sciatic nerve section. mGluR5-IR plotted against somata cross-sectional area in naïve target-specific population $(a)$ (backlabeled with FB from the skin of the hindpaw), the ipsilateral damaged population (b) (labeled using FR introduced to the cut end of the sciatic nerve), and the population of ipsilateral DRG neurons left intact (c) (labeled by injecting FB into the skin of the hindpaw after the sciatic nerve surgery). $d$ is a picture of the sciatic nerve at the point of partial section that has been stained for mGluR5. mGluR5-IR is increased in the damaged portion of the nerve (indicated by an arrow).e, PI index of mGluR5-IR in the target-specific naïve and ipsilateral damaged and intact neuronal populations. Data were compared using Fisher's exact test, and no significant differences were found in mGluR5-IR between these populations. Scale bar, $200 \mu \mathrm{m}$.

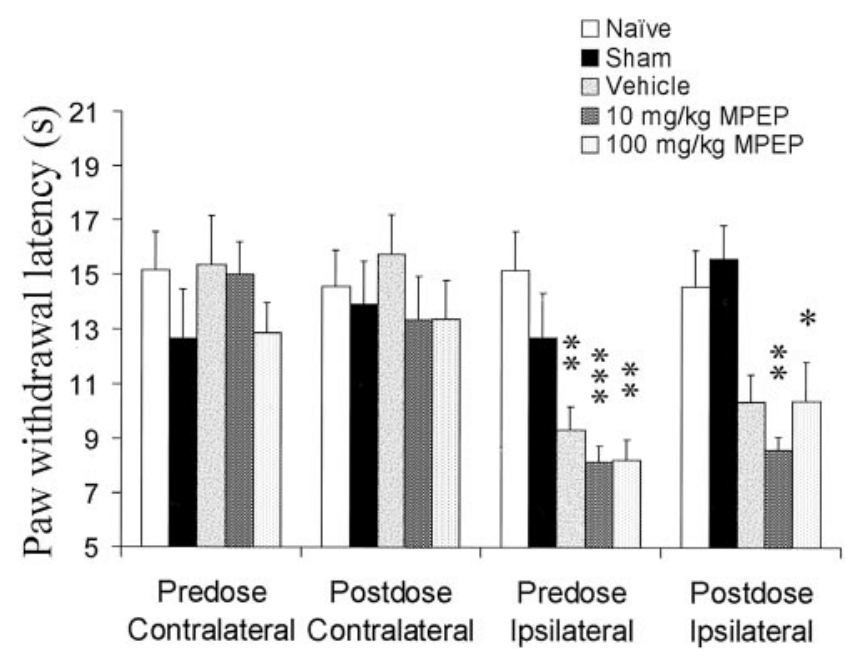

Figure 5. The effects of MPEP, a specific mGluR5 antagonist on the thermal hyperalgesia resulting from partial sciatic nerve section. Paw withdrawal latencies were measured 2 weeks after the partial sciatic nerve section. No sham effects were found at this time, and all ipsilateral paws before drug administration showed significant thermal hyperalgesia $(p<$ 0.01). Three hours after oral administration of MPEP (at 10 and 100 $\mathrm{mg} / \mathrm{kg}$ ), thermal hyperalgesia was remeasured. MPEP had no effects on the paw withdrawal latency of naïve or sham-operated rats. The hyperalgesia of both of the MPEP-treated groups that had had partial nerve section was not reversed and was still significant compared with control groups. Data were compared using a parametric ANOVA with Tukey's post hoc test $(p<0.05) . n=12$; data from two separate experiments were combined.

study, thermal hyperalgesia was not reversed by systemic administration of MPEP (10 or $100 \mathrm{mg} / \mathrm{kg}$, p.o.).

In another neuropathic model, in which the L5 spinal nerve is ligated, large increases in mGluR5-IR in both uninjured L4 and injured L5 ipsilateral DRG were measured compared with contralateral DRG. Significant mechanical and thermal hyperalgesia, as well as allodynia, was measured 3 weeks after the surgery. Systemic administration of MPEP did not affect either mechanical hyperalgesia or allodynia; however, a dose-dependent increase in paw withdrawal latency to a thermal stimulus was measured. This agrees with published data using a different mGluR5 antagonist, SIB-1757 (Dogrul et al., 2000). Hence, we show a correlation between increased mGluR5-IR and the ability of the mGluR5 agonist MPEP to demonstrate anti-hyperalgesic activity, at least against thermal hyperalgesia.

One possibility that we have not explored is that mGluR5 and MPEP sensitivity in the sciatic nerve ligation model is late developing. We examined only our standard 2 week time point. However, in the spinal nerve ligation model, time points as early as $5 \mathrm{~d}$ (Dogrul et al. 2000) and as late as 3 weeks (this study) after surgery show reversal of thermal hyperalgesia by mGluR5 antagonists.

We used the A-fiber marker N52 to further investigate the neuronal populations involved and found that all of these increases in mGluR5 after L5 spinal nerve lesion were in the myelinated A-fiber cell bodies. Furthermore, many of the of A-fibers expressing mGluR5 after spinal nerve ligation also express VR1 after such injury. Although there is currently no supportive evidence, it is interesting to speculate that mGluR5 and VR1 are coupled in these neurons and that inhibition of mGluR5 activation in turn inhibits VR1 opening and thermal hyperalgesia.

We showed that MPEP reverses the thermal hyperalgesia that develops after L5 spinal nerve ligation, and this correlates with a large increase in mGluR5-IR in the ipsilateral A-fibers of L4 and L5 DRG. However, thermal hyperalgesia is conventionally thought to be mediated by nonmyelinated C-fibers (Shir and 
Figure 6. mGluR5 expression was measured 3 weeks after L5 spinal nerve ligation. $a-c$ are scatter plots of contralateral, ipsilateral L4 (intact), and ipsilateral L5 (ligated) mGluR5-IR, respectively. $d$ shows that the PI index of mGluR5 expression has increased in the intact L4 ipsilateral DRG and in the L5-ligated DRG compared with contralaterals $(p<$ 0.001). $e$ examines these differences in more detail using anti-neurofilament to separate the A- and $\mathrm{C}$-fiber populations. Interestingly, there are large, significant increases in mGluR5 expression in the A-fibers of both L4 and L5 ipsilateral DRG neuronal populations, with no change detected in the $\mathrm{C}$-fiber population. Data were compared using Fisher's exact test; ${ }^{* * *} p<0.001$.

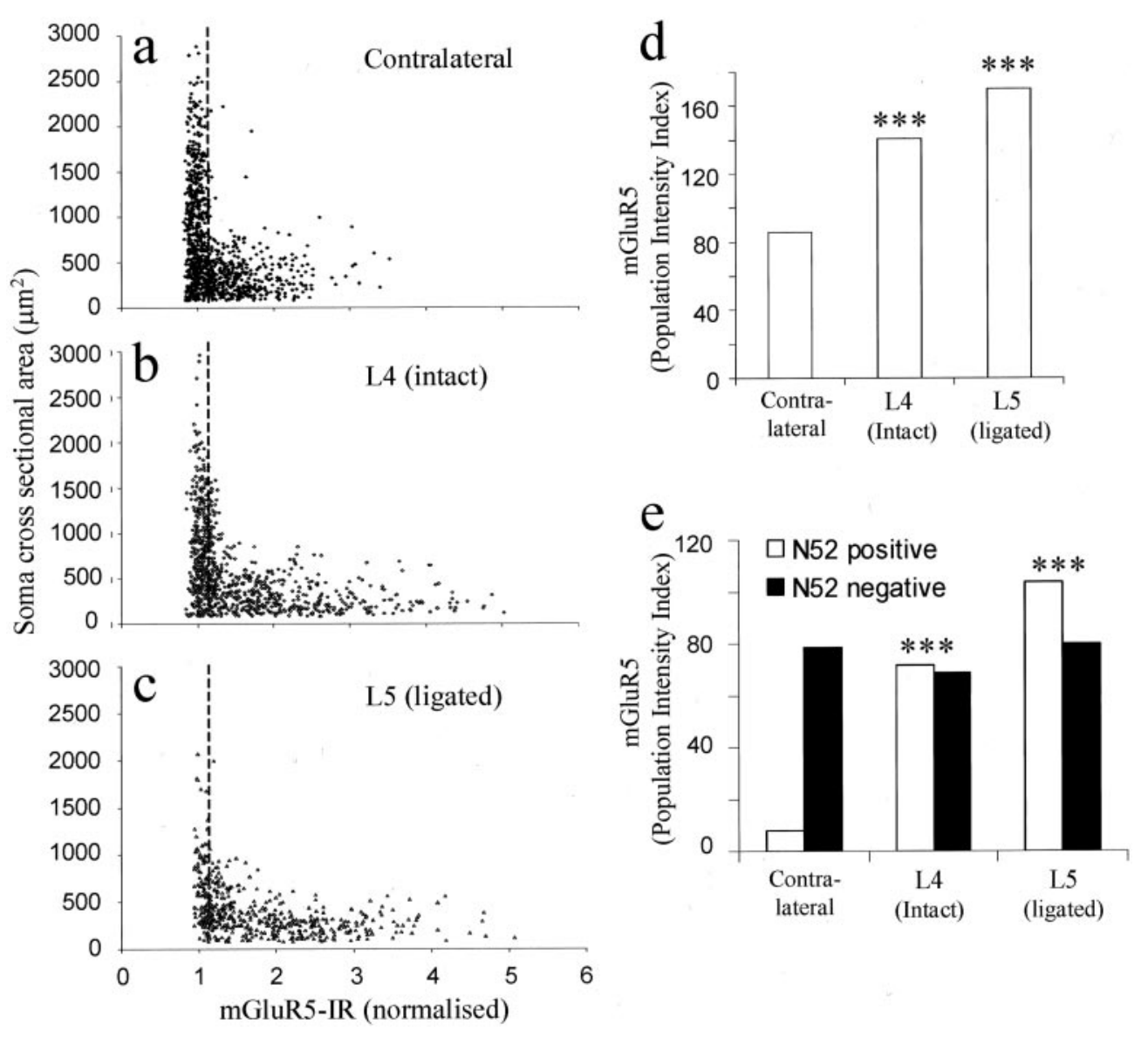

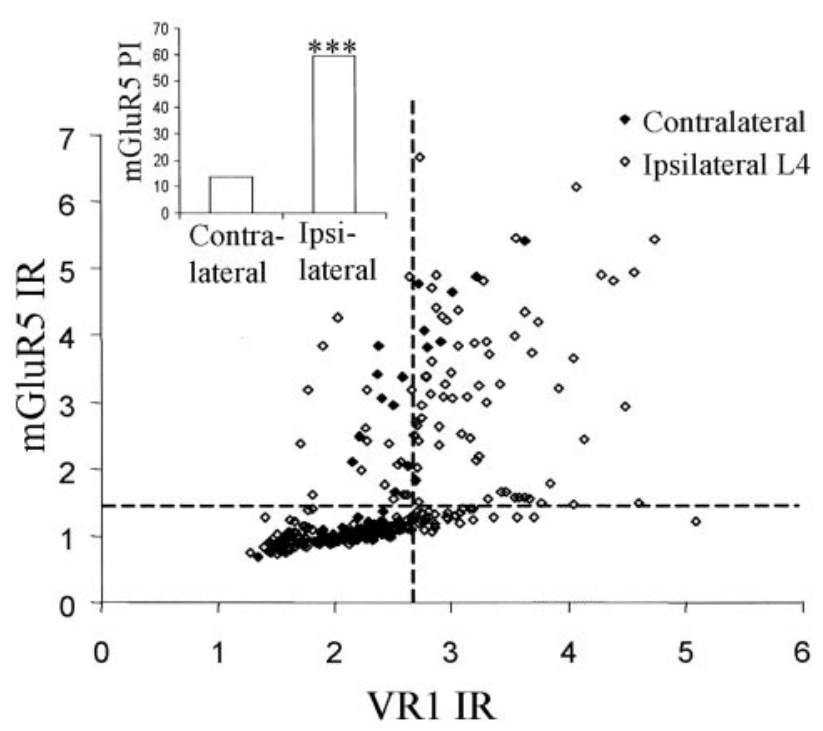

Figure 7. Three weeks after L5 spinal nerve ligation, mGluR5 expression was colocalized with VR1 expression in myelinated A-fibers, which were identified using an anti-neurofilament stain. The scatter plot shows levels of VR1 and mGluR5 on A-fibers in ipsilateral and contralateral L4 DRG after L5 spinal nerve ligation. The cutoff lines were determined for both VR1- and mGluR5-positive staining, as described in Materials and Methods. Neurons in the top right-hand quadrant are positive for both mGluR5 and VR1 and are almost absent in the contralateral DRG.
Seltzer, 1990; Vatine et al., 1998; Ossipov et al., 1999). Indeed, tactile allodynia is mediated through A-fibers (Cervero and Laird, 1996), but, despite large increases in mGluR5 expression in the ipsilateral myelinated somata after spinal nerve injury, MPEP failed to reverse the tactile allodynia produced. Tools such as capsaicin and resiniferatoxin (RTX) have been widely used to characterize $\mathrm{C}$-fiber-mediated sensation. Capsaicin produces a long-lasting desensitization of the fibers on which its receptor VR1 is located (Szallasi et al., 1989), with RTX, a more potent VR1 agonist, producing a longer-lasting desensitization (Craft et al., 1995). Peripheral RTX treatment of rats can induce $>4$ weeks of thermal hypoalgesia with no effect on mechanical thresholds (Xu et al., 1997). This and other evidence supports the involvement of C-fibers in the mediation of thermal nociception (Shir and Seltzer, 1990; Vatine et al., 1998). Ossipov et al. (1999) found that RTX treatment after L5 spinal nerve ligation resulted in a significant reversal of the thermal hyperalgesia associated with this nerve injury, supporting the idea that VR1 acts as a transducer for noxious heat (Caterina et al., 1997). However, after similar nerve injury, we showed that the number of VR1-positive A-fiber somata increases to $33 \%$ in ipsilateral L4 DRG neurons from just $3 \%$ in naïve rats (Hudson et al. 2001). Hence, after such nerve injury, treatment with capsaicin or RTX may desensitize up to $33 \%$ of A-fibers, as well as the small-diameter C-fiber population. Together, these results suggest that capsaicin or RTX treatment may be useful tools in examining the function of VR1positive neurons; however, after nerve injury, these are unlikely to be present solely on unmyelinated $\mathrm{C}$-fibers.

The notion that A-fibers may mediate pain after nerve injury is not a new one. Indeed, some A-fibers are now thought to be 


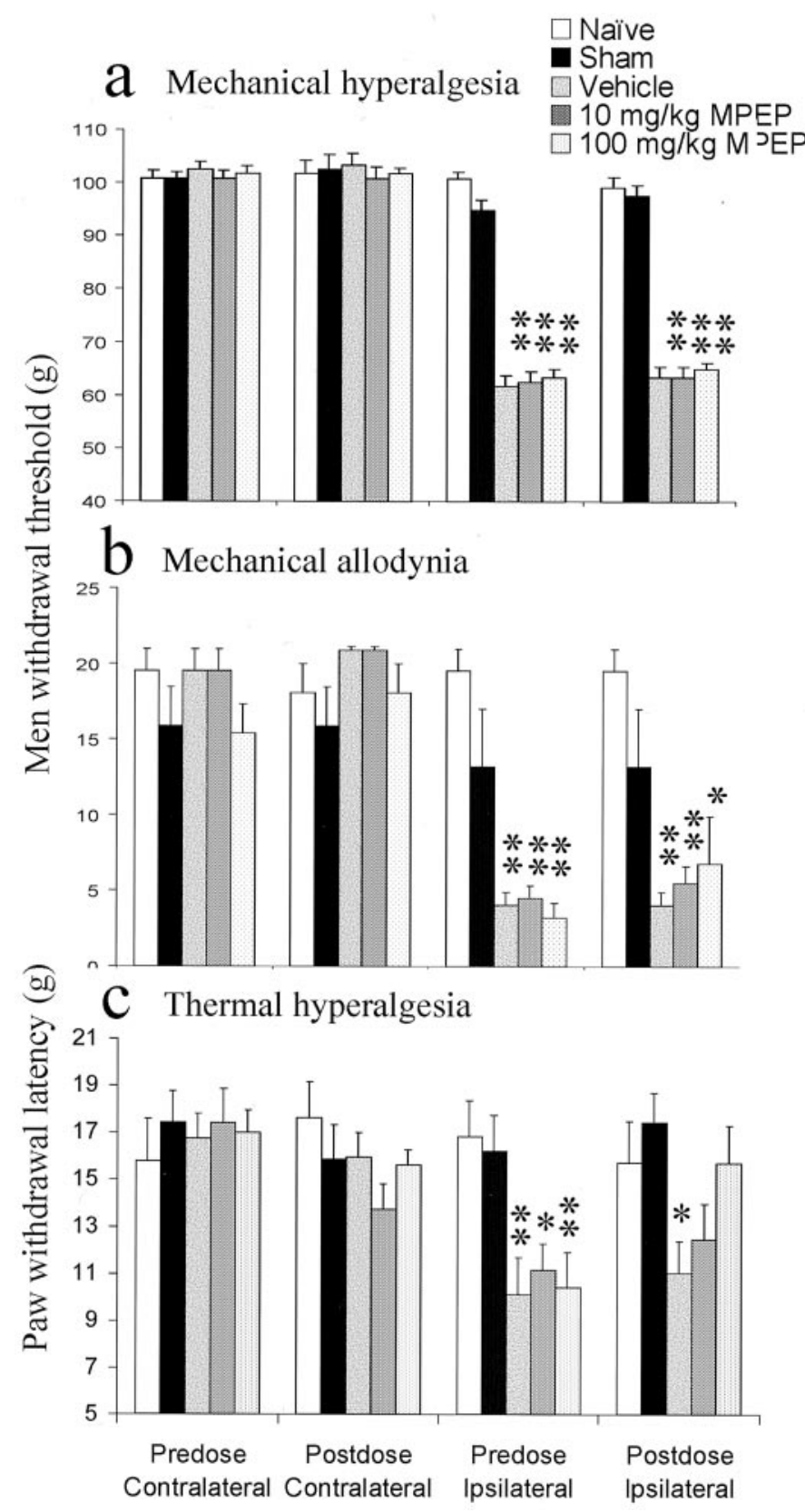

Figure 8. The effect of MPEP on the behavioral responses induced by L5 spinal nerve ligation was examined. Three weeks after surgery, shamoperated animals displayed paw withdrawal latencies similar to those of naïve animals in all modalities measured. $a$, Mechanical hyperalgesia was measured after L5 spinal nerve ligation, and the effects of MPEP were determined. Significant mechanical hyperalgesia was measured in all animals after L5 spinal nerve ligation. MPEP failed to reverse the induced mechanical hyperalgesia. $b$, Significant mechanical allodynia developed in all rats after L5 spinal nerve ligation. Systemic administration of MPEP failed to reverse this mechanical allodynia. $c$, Before the administration of MPEP, all ipsilateral spinal nerve-ligated paws exhibited significant thermal hyperalgesia. Oral administration of MPEP $(10$ and $100 \mathrm{mg} / \mathrm{kg}$ ) produced a dose-dependent antihyperalgesic effect. At $100 \mathrm{mg} / \mathrm{kg}$, paw withdrawal latencies of spinal nerve-lesioned paws to a thermal stimulus were back to naïve levels. Data were compared using a parametric ANOVA with Tukey's post hoc test; *p $<0.05 ;{ }^{* *} p<0.01 . n=6$ for each condition. nociceptive in naïve animals (Fang et al., 2000), although their role in pain after nerve damage may be the result of anatomical rearrangements. After peripheral nerve injury, myelinated A-fibers, which usually terminate in lamina III of the dorsal horn and transmit non-noxious stimuli, have been shown to form collateral sprouts into lamina II of the dorsal horn (Woolf et al., 1992; Lekan et al., 1996). In the cat, such collateral sprouts are maintained for up to 1 year after the nerve injury (Koerber et al., 1994). Indeed, using intracellular recording from spinal cord slices, these sprouts into lamina II have been shown to establish functional contacts with dorsal horn neurons that would normally receive inputs from $\mathrm{A} \delta$ - or $\mathrm{C}$-fibers (Kohama et al., 2000). This could explain the hypersensitivity seen after nerve injury in which normally innocuous stimuli cause a noxious response.

This study has revealed differences in mGluR5 expression levels in different nerve injury models; the reasons for this are as yet unclear. There are differences between spinal nerve ligation and partial nerve section, the main one being that, after spinal nerve ligation, the somata of damaged and intact neurons are anatomically distinct. After partial nerve section, however, damaged and intact populations are adjacent within the same ganglia. The interaction between these adjacent intact and damaged ganglia could play a role in the development of nerve injury-induced pain. Indeed, it has been shown that, after nerve section, adjacent intact and damaged DRG somata communicate by cross excitation (Devor and Wall, 1990). There is current controversy as to the relative importance of damaged and intact primary afferent fibers after nerve injury. We showed previously that, although VR1 decreased in the damaged population after both sciatic nerve partial section and L5 spinal nerve ligation, large increases were measured in the population left intact (Hudson et al., 2001). However, here we found that mGluR5 expression was unchanged in both intact and damaged populations after partial sciatic nerve injury but increased in both the damaged and undamaged ganglia after L5 spinal nerve ligation. Thus, mGluR5 and VR1 are likely to be under different regulatory controls.

In the current study, mechanical and thermal hyperalgesia, as well as allodynia, developed in two models of neuropathic pain, i.e., spinal nerve ligation or partial sciatic nerve section. Oral administration of MPEP dose dependently reversed the thermal hyperalgesia after spinal nerve ligation but not after partial nerve section. Furthermore, it was only after spinal nerve ligation that increases in mGluR5 were measured in the DRG neurons. Detailed analysis revealed that the majority of the increases measured in mGluR5 occurred in the A-fiber neuronal population, suggesting a new role for these myelinated afferents in thermal hyperalgesia.

\section{REFERENCES}

Baranauskas G, Nistri A (1998) Sensitization of pain pathways in the spinal cord: cellular mechanisms. Prog Neurobiol 54:349-365.

Bhave G, Karim F, Carlton SM, Gereau RW (2001) Peripheral group I metabotropic glutamate receptors modulate nociception in mice. Nat Neurosci 4:417-423.

Caterina MJ, Schumacher MA, Tominaga M, Rosen TA, Levine JD, Julius D (1997) The capsaicin receptor: a heat-activated ion channel in the pain pathway. Nature 389:816-824

Cervero F, Laird JM (1996) Mechanisms of allodynia: interactions between sensitive mechanoreceptors and nociceptors. NeuroReport 7:526-528.

Craft RM, Cohen SM, Porreca F (1995) Long-lasting desensitization of bladder afferents following intravesical resiniferatoxin and capsaicin in the rat. Pain 61:317-323.

Devor M, Wall PD (1990) Cross-excitation in dorsal root ganglia of nerve-injured and intact rats. J Neurophysiol 64:1733-1746.

Dogrul A, Ossipov MH, Lai J, Malan Jr TP, Porreca F (2000) Peripheral 
and spinal antihyperalgesic activity of SIB-1757, a metabotropic glutamate receptor (mGLUR(5)) antagonist, in experimental neuropathic pain in rats. Neurosci Lett 292:115-118.

Fang X, Djouhri L, Lawson SJ (2000) Sensory receptor type and conduction velocity of A-fibre dorsal root ganglion neurons that express TrkA in the rat in vivo. Eur J Neurosci 12:484.

Gasparini F, Lingenhohl K, Stoehr N, Flor PJ, Heinrich M, Vranesic I, Biollaz M, Allgeier H, Heckendorn R, Urwyler S, Varney MA, Johnson EC, Hess SD, Rao SP, Sacaan AI, Santori EM, Velicelebi G, Kuhn R (1999) 2-Methyl-6-(phenylethynyl)-pyridine (MPEP), a potent, selective and systemically active mGlu5 receptor antagonist. Neuropharmacology 38:1493-1503.

Gold MA (2000) Spinal nerve ligation: what to blame for the pain and why. Pain 84:117-120.

Hudson LJ, Bevan S, Wotherspoon G, Gentry C, Fox A, Winter J (2001) VR1 protein expression increases in undamaged DRG neurons after partial nerve injury. Eur J Neurosci 13:2105-2114

Kim SH, Chung JM (1992) An experimental model for peripheral neuropathy produced by segmental spinal nerve ligation in the rat. Pain 50:355-363.

Koerber HR, Mirnics K, Brown PB, Mendell LM (1994) Central sprouting and functional plasticity of regenerated primary afferents. J Neurosci 14:3655-3671.

Kohama I, Ishikawa K, Kocsis JD (2000) Synaptic reorganization in the substantia gelatinosa after peripheral nerve neuroma formation: aberrant innervation of lamina II neurons by Abeta afferents. J Neurosci 20:1538-1549.

Lee BH, Yoon YW, Chung K, Chung JM (1998) Comparison of sympathetic sprouting in sensory ganglia in three animal models of neuropathic pain. Exp Brain Res 120:432-438.

Lekan HA, Carlton SM, Coggeshall RE (1996) Sprouting of A beta fibers into lamina II of the rat dorsal horn in peripheral neuropathy. Neurosci Lett 208:147-150.

Ossipov MH, Bian D, Malan TPJ, Lai J, Porreca F (1999) Lack of involvement of capsaicin-sensitive primary afferents in nerve-ligation injury induced tactile allodynia in rats. Pain 79:127-133.

Petersen-Zeitz KR, Basbaum AI (1999) Second messengers, the sub- stantia gelatinosa and injury-induced persistent pain. Pain [Suppl] 6:S5-S12.

Shir Y, Seltzer Z (1990) A-fibers mediate mechanical hyperesthesia and allodynia and $\mathrm{C}$-fibers mediate thermal hyperalgesia in a new model of causalgiform pain disorders in rats. Neurosci Lett 115:62-67.

Szallasi A, Joo F, Blumberg PM (1989) Duration of desensitization and ultrastructural changes in dorsal root ganglia in rats treated with resiniferatoxin, an ultrapotent capsaicin analog. Brain Res 503:68-72.

Urban MO, Gebhart GF (1999) Supraspinal contributions to hyperalgesia. Proc Natl Acad Sci USA 96:7687-7692.

Valerio A, Paterlini M, Boifava M, Memo M, Spano P (1997a) Metabotropic glutamate receptor mRNA expression in rat spinal cord. NeuroReport 8:2695-2699.

Valerio A, Rizzonelli P, Paterlini M, Moretto G, Knopfel T, Kuhn R, Memo M, Spano P (1997b) mGluR5 metabotropic glutamate receptor distribution in rat and human spinal cord: a developmental study. Neurosci Res 28:49-57.

Vatine JJ, Argov R, Seltzer Z (1998) Brief electrical stimulation of c-fibers in rats produces thermal hyperalgesia lasting weeks. Neurosci Lett 246:125-128.

Walker K, Bowes M, Panesar M, Davis A, Gentry C, Kesingland A, Gasparini F, Spooren W, Stoehr N, Pagano A, Flor PJ, Vranesic I, Lingenhoehl K, Johnson EC, Varney M, Urban L, Kuhn R (2001a) Metabotropic glutamate receptor subtype 5 (mGlu5) and nociceptive function. I. Selective blockade of mGlu5 receptors in models of acute persistent and chronic pain. Neuropharmacology 40:1-9.

Walker K, Reeve A, Bowes M, Winter J, Wotherspoon G, Davis A, Schmid P, Gasparini F, Kuhn R, Urban L (2001b) mGlu5 receptors, nociceptive function II. mGlu5 receptors functionally expressed on peripheral sensory neurones mediate inflammatory hyperalgesia Neuropharmacology 40:10-19.

Woolf CJ, Shortland P, Coggeshall RE (1992) Peripheral nerve injury triggers central sprouting of myelinated afferents. Nature 355:75-78.

Xu XJ, Farkas-Szallasi T, Lundberg JM, Hokfelt T, Wiesenfeld-Hallin Z, Szallasi A (1997) Effects of the capsaicin analogue resiniferatoxin on spinal nociceptive mechanisms in the rat: behavioral, electrophysiological and in situ hybridization studies. Brain Res 752:52-60. 